\title{
How We Do It: Optimizing the Histological Mapping of Thin Delicate Tissue in Mohs Micrographic Surgery-The "Paper Cut Technique"
}

Michael Kunz, MD; Lauren Poynter, HT; Kimberly Walker, HT; Ally-Khan Somani, MD $\mathrm{PhD}$.

Department of Dermatology Indiana University School of Medicine Indianapolis, Indiana 
Hallmarks of Mohs micrographic surgery (MMS) are precise and complete surgical margin control to achieve high cure rates for locally aggressive skin tumors and provision of maximum tissue preservation..$^{1,2}$ Various techniques have been shown to be useful for tissue resection, orientation, transfer, flattening, inking, mapping, and processing, and adaptations to the routine procedure have been suggested for small or fragile specimens. $^{3,4}$

Accurate handling of thin delicate layers (i.e., fascia, perichondrium, periosteum, or thin muscle) can be challenging because this tissue is inherently fragile and prone to desiccation and shrinkage. This can lead to tissue distortion during tissue transfer for histological processing and ultimately yield imprecision in tissue mapping. In this article, we present a simple and novel method to facilitate accurate tissue mapping of delicate Mohs layers, which we call the "Paper Cut Technique" (PCT).

Methods During MMS when deep margins are found to be positive for tumor and it is necessary to procure additional thin Mohs layers, the PCT can be used. The deep tissue is first inked with dye to ensure complete removal. ${ }^{5}$ After hydrodissection, the thin tissue layer is excised. The specimen (prone to desiccation and contraction) is then immediately placed on filter paper (Figure 1) where it can be flattened and stretched while it is still moist. An accurate map is drawn, and the specimen is inked directly on the paper, preserving its configuration and orientation. In the laboratory, the redundant part of the paper is trimmed off with a scalpel blade or scissors, leaving behind paper attached to the entire deep margin of the removed tissue (Figure 2A). The specimen along with the attached paper is then processed for cryosectioning in the usual manner without the need for additional tissue manipulation. First, it is frozen to the plate where it lays flat (Figure $2 \mathrm{~B}$ ), and then it is embedded in optimal cutting temperature compound (Figure $2 \mathrm{C}$ ) before it gets frozen onto the chuck. The initial sections of the block consist of paper and are discarded until the blade makes contact with the target tissue to obtain the true marginal section.

Figure 1. Thin deep fascial/periosteal layer oriented and placed on filter paper.

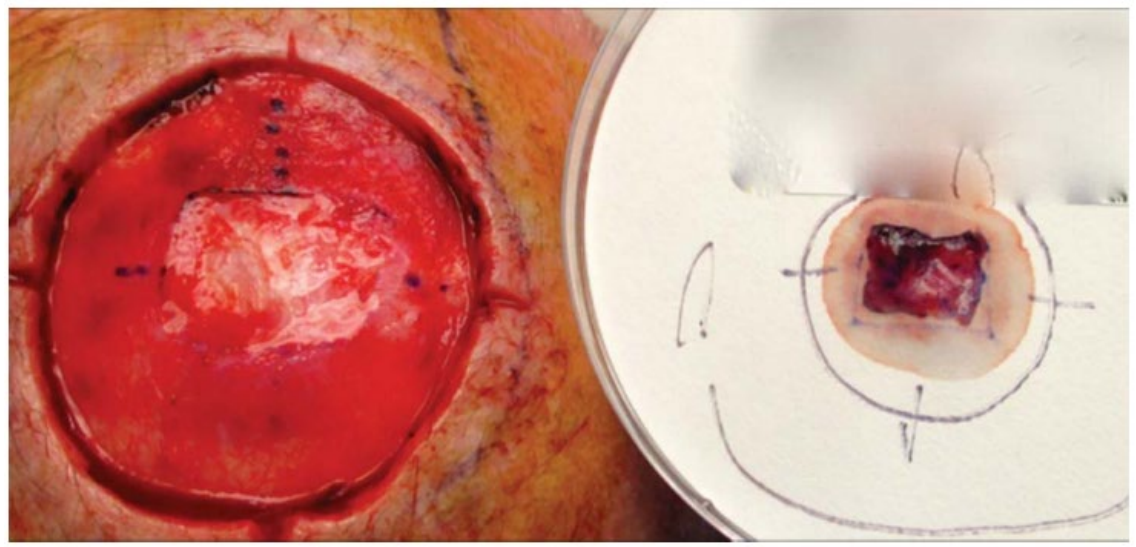


Figure 2. Processing the tissue stuck on paper: $(A)$ Colored tissue with trimmed paper attached to its deep margin. (B) Placement of the tissue-paper combination on the plate. (C) Application of optimal cutting temperature compound.

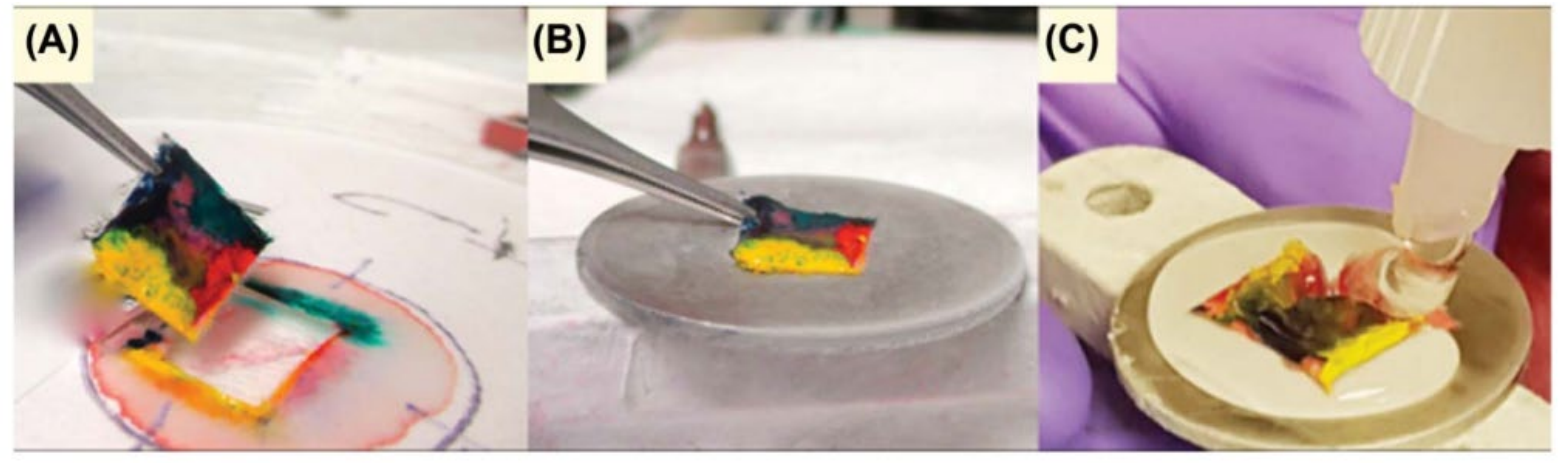

Figure 3. (A) Paper remnant on paper cut histological frozen section, magnification $\times 400$. (B) Paper cut histological frozen section with complete colored edges, overview, magnification $\times 20$.

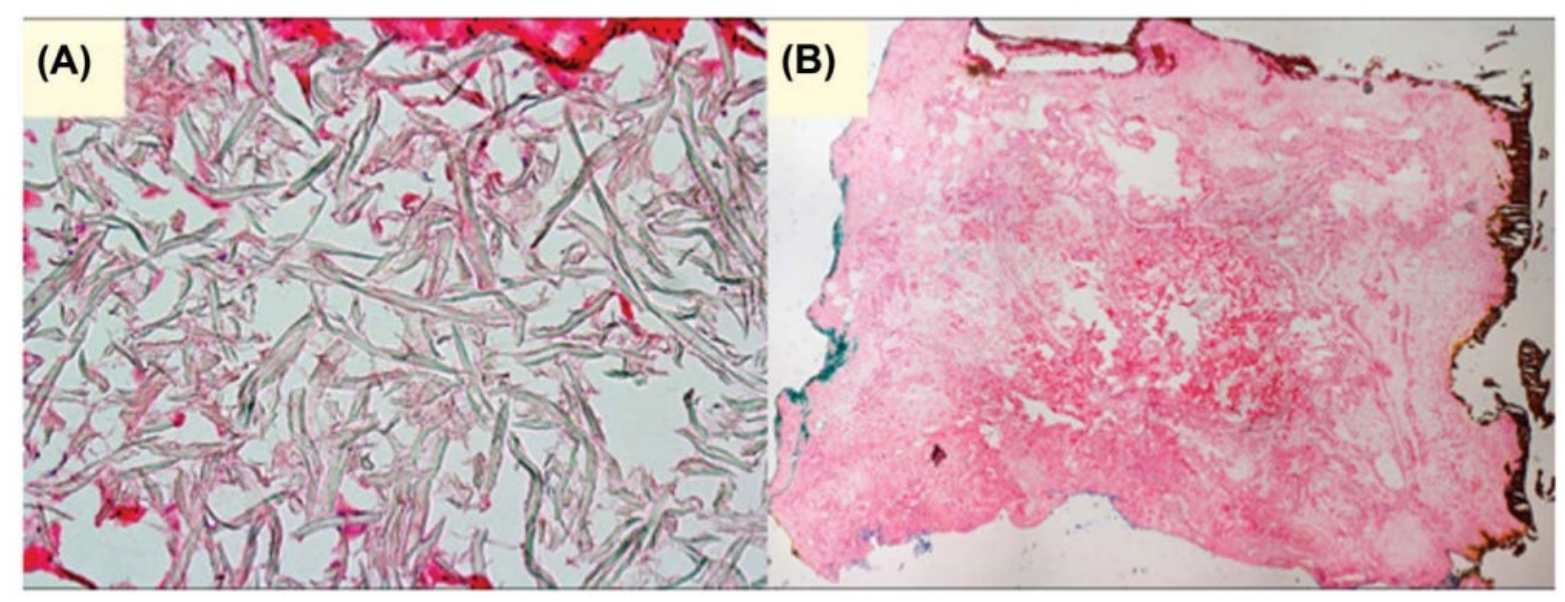

\section{RESULTS}

From April 2018 until May 2019, a total of 14 thin delicate Mohs layers were successfully processed with the PCT. All tissue layers subjected to PCT were obtained from patients undergoing MMS for nonmelanoma skin cancer (basal cell carcinoma and squamous cell carcinoma) or dermatofibrosarcoma protuberans. In all cases, the PCT maintained the configuration of the procured tissue with minimal to no distortion, ensuring accurate histological mapping. We further tested several paper sources (Whatman Grade $3 \mathrm{MM}$ CHR filter paper, Fisherbrand FilterPaper, Standard Laser Printer paper Xerographic 8.5x11", Kimwipes KIMTECH Delicate Task Wipers and Reynolds Cut-Rite Wax Paper, Kimberly-Clark Professional, Reynolds Consumer Products) for the PCT. The paper was rated by our histology technicians according to its absorptive capacity, tissue adherence, stability, thickness (number of sections to reach block), and ease of cryosectioning. We found regular Petri dish filter paper (Fisherbrand Filter Paper, Qualitative P4, Porosity Medium-Fine, Flow Rate: Slow; Fisher Scientific, Hampton, NH) to perform the best 
overall. A thickness of $210 \mu \mathrm{m}$ had to be removed of this paper to obtain a full tissue section. Histologically, paper fibers are readily distinguished from other structure found on skin, subcutaneous tissue, fascia, or tumors (Figure 3A). No increased effects of usage such as dullness or nicking of the blade were noticed when cutting through filter paper. The PCT has been successfully used with great reproducibility for layers taken from scalp and maxillary periosteum, auricular perichondrium as well as fascia of variable sites: temporalis muscle, orbicularis oculi muscle, orbicularis oris muscle, zygomaticus major/minor muscles, and fascia of dorsal hand and ventral forearm (Figure 3B). The quality of histological slides was excellent for all types of tumors included.

\section{CONCLUSION}

Advantages of the PCT are minimization of tissue manipulation by avoiding the need for tissue transfer during histological processing. This technique facilitates the reliable production of high-quality histological slides and retains the accurate micrographic mapping of thin delicate tissues normally prone to desiccation, shrinkage, and distortion. Furthermore, during the alignment and facing of the block, paper is being cut off instead of tissue. This will result in the minimization of false positives and the production of a complete true marginal section. We posit that the drawbacks of the PCT are negligible. The filter paper needs to be ready on the tray before starting the procedure, and an additional step (positioning of the thin and delicate tissue layer on the paper and trimming the paper after color mapping) is added resulting in a slightly prolonged operation time for the Mohs surgeon. Regular Petri dish filter paper is readily available and inexpensive, so the added cost is insignificant. No change of equipment is necessary and training of histology technicians is quick and easy. In summary, the PCT is a novel innovative method that can be readily incorporated into the daily routine of MMS for selected cases.

\section{REFERENCES}

1. Shriner DL, McCoy DK, Goldberg DJ, Wagner RF Jr. Mohs micrographic surgery. J Am Acad Dermatol 1998;39:79-97.

2. Lee E, Wolverton JE, Somani AK. A simple, effective analogy to elucidate the Mohs micrographic surgery procedure-the peanut butter cup. JAMA Dermatol 2017;153:743-4.

3. Li JY, Silapunt S, Migden MR, McGinness JL, et al. Mohs mapping fidelity: optimizing orientation, accuracy, and tissue identification in Mohs surgery. Dermatol Surg 2018;44:1-9.

4. Kist DA, Zachary CB. Direct transfer of curettings to cryostat chuck in Mohs micrographic surgery. J Dermatol Surg Oncol 1992;18:226-8.

5. McCallister M, Krishnan RS, Desai RS, Krathen R, et al. Obtaining thin and complete Mohs layers using the methylene blue staining technique. Dermatol Surg 2011;37:10267. 